\title{
Research on a Scheduling Mechanism in a Complex System Based on TOC
}

\author{
Zhang Wen ${ }^{\mathrm{a}, \mathrm{b}}$ Zhang Ya-Ming ${ }^{\mathrm{a}}$,Chen Jinbo ${ }^{\mathrm{c}}$ Leng Kaijun ${ }^{\mathrm{c}}$ \\ ( ${ }^{a}$.School of Economics \& Management, Yanshan University, Qinhuangdao, P.R.China, 066004) \\ $\left({ }^{b}\right.$.Gannan Normal University,Ganzhou,P.R.China,341000) \\ ( ${ }^{c}$.Research Center of Hubei Logistics development, Hubei University of \\ Economics, Wuhan,P.R.China,430205)
}

\begin{abstract}
Under the condition where there is no seasonal demand fluctuation, short life cycle product supply chain should confront the market environment such as the decreasing of product value, the launch of substitutes and the appearance of competitors' similar products, and the supply chain will become a very complex system. In this paper, the authors consider a TOC-based scheduling mechanism in this complex supply chain system. under the constant total production cost, it is more important to improve the availability of the wanted product in order to enhance the overall supply chain competitiveness so to obtain more effective output( profit rate) for the supply chain in a long period.Especially we try to apply the SDBR concept into a schedule mechanism in a particular supply chain system, and use numerical analysis to test the efficiency of the proposed method.
\end{abstract}

\section{Introduction}

The complexity and dynamism that characterize the context in which companies operate nowadays have drawn a new competitive environment. In it, the development of information technologies, the decrease in transport costs and the breaking down of barriers between markets, among other reasons, have led to the perception that competition between companies is no longer constrained to the product itself, but it goes much further. For this reason, the concept of Supply Chain Management (SCM) has gained a lot of strength to the point of having a strategic importance. The current global economic crisis, consequence of many relevant systemic factors due to the fact that globalization still has not been able to develop systemic dynamic properties to deal with a growing variety of requirements, is creating conditions which increase awareness to adopt new approaches to make business (among others, Schweitzer et al., 2009); hence, SCM is a boiling area for innovation.

Analyzing the supply chain, Forrester (1961) noted that changes in demand are significantly amplified along the system, as orders move away from the client. It was called the Bullwhip Effect. He studied the problem from the perspective of system dynamics. This amplification is also evidenced in the famous 'Beer Game' (Sterman, 1989), which shows the complexity of SCM. He concluded that the Bullwhip Effect is generated from local-optimal solutions adopted by supply chain members. This can be considered as a major cause of inefficiencies in the supply chain (Disney, Farasyn, Lambrecht, 
Towill, \& Van de Velde, 2005), because it tends to increase storage, labor, inventory, shortage and transport costs. Lee, Padmanabhan, and Whang (1997) identified four root causes in the generation of Bullwhip Effect in supply chains: (1) wrong demand forecasting; (2) grouping of orders into batches; (3) fluctuation in the products prices; and (4) corporate policies regarding shortage. The same idea underlies behind all of them: the transmission of faulty information to the supply chain. Therefore, the first approaches in the search for a solution to this problem were based on trying to coordinate the supply chain. Some practices that have been successfully implemented in companies are Vendor Managed Inventory (Andel, 1996), Efficient Consumer Response (McKinsey, 1992) and Collaborative Planning, Forecasting and Replenishment (DesMarteu, 1998). Nevertheless, the Bullwhip Effect is still a major concern around operations management in the supply chain. Chen and Lee (2012) discussed the linkage between the bullwhip measure and the supply chain cost performance, capturing the essence of most real-world scenarios.

Under the condition where there is no seasonal demand fluctuation, short life cycle product supply chain should confront the market environment such as the decreasing of product value, the launch of substitutes and the appearance of competitors' similar products, and the supply chain will become a very complex system. As a result, the ability to provide a proper supply chain coordination method in general condition to improve its competitive advantage and more profit is one of the key research point of supply chain coordination study. At the moment, most supply and demand decision methods of this kind, its common solution is taking the classical method MTO ( Make to order), However, under this strategy, the biggest issue is there is no extra capacity left for the manufacturer to make other decision. Because of the fixed time of delivery, the manufacturer has to devote every possibility to produce, even put more cost in order to guarantee the delivery which cause many coordination problems during the process.

In this paper, the author consider a TOC-based scheduling mechanism in this complex supply chain system. under the constant total production cost, it is more important to improve the availability of the wanted product in order to enhance the overall supply chain competitiveness so to obtain more effective output( profit rate) for the supply chain in a long period. Therefore, the basis of the the supply chain coordination is to fist apply the VMI replenishment system in every link, then adopt Making to Avaliability (MTA)method to coordinate the supply chain, which the distributor understands the client's demand and sends production oder to the manufacturer to produce part of the products in advance while negotiating with the clients. The manufacturer can follow a retention capacity of production in advance, once 
the sales order was signed, the manufacturer can deliver part of the products in advance through VMI replenishment system. Thus, the distributor can not only guarantee the time of delivery, but also sell the products with a higher price during the negotiation. Ultimately, it will bring higher effective output for the whole supply chain.

\section{Literature Review}

In the book 'The Goal-A Process of Ongoing Improvement' (1984) Elihayu M. Goldratt described his view about the best way to manage a company. He did it through fiction, telling how a troubled company managed to get over this situation. In a subsequent scientific work, Goldratt (1990) presented the Theory of Constraints (TOC) in more detail. This theory comprises three interrelated areas (Simatupang, Hurley, \& Evans, 1997): logistics, logical thinking and performance measurement. In logistics, the methodology is based on the DBR scheduling method (Goldratt \& Cox, 1984). The logical thinking is based on a continuous improvement cycle with five steps: (I) Identify the bottleneck; (II) Decide how to exploit the bottleneck; (III) Subordinate everything else in the system to the previous step; (IV) Elevate the bottleneck; and (V) Evaluate if the bottleneck has been broken, and return to the beginning. The performance measurement, which quantifies the application of this methodology, encompasses operational measures (throughput, inventory and operating expense) and financial measures (net profit, return on investment and cash flow), which obey to the same view: the only goal of the organization is to make money now and in the future.

Although TOC was initially oriented on the production system of the company, its application to other areas of the business has been proposed, such as marketing and sales (Goldratt, 1994), project management (Goldratt, 1997) or SCM (Goldratt, Schragenheim, \& Ptak, 2000). In this latter area, several authors have researched the application of the TOC. As an example, Umble, and von Deylen (2001) described the application of TOC in the implementation of an ERP system to manage the supply chain. Cox and Spencer (1998) proposed a method for SCM through TOC, valid when one company directs the entire chain. However, when this assumption does not apply and there are different companies in the same supply chain, the implementation of TOC is more complex. A dilemma rises because each company has to decide between gearing to the interests of the supply chain as a whole and pursuing only their own interests. Simatupang, Wright, and Sridharan (2004) showed that collaboration between different independent firms, according to the TOC, generates a much larger benefits to participants than the consideration of individual interests of each company. $\mathrm{Wu}$, Chen, Tsai, and Tsai (2010) developed an enhanced simulation replenishment model for TOC-SCRS (Theory of Constraints-Supply Chain Replenishment System) under capacity constraint in the different levels. The TOC-SCRS (Yuan, Chang, \& Li, 2003) is a methodology widely used in 
businesses nowadays to improve the SCM and to reduce Bullwhip Effect. It is based on the use of two strategies (Cole \& Jacob, 2003): (I) Each node holds enough stock to cover demand during the time it takes to replenish reliably; and (II) Each node orders only to replenish what was sold. The authors demonstrated the effectiveness of this system, in solving the conflict generated in determining the frequency and quantity of replenishment when the TOC-SCRS is applied in a plant or a central warehouse. In a later work (Wu, Lee, \& Tsai, 2014), they proposed a two-level replenishment frequency model for the TOC-SCRS under the same constraints, which is especially suitable to a plan in which different products have a large sales volume variation. This methodology facilitates a plant or a central warehouse the implementation of TOC-SCRS.

Furthermore, hundreds of DBR successful stories have been reportedand these reports have claimed that highly reliable DDP can be rapidly achieved (Mabin \& Balderstone, 2000; Umble, Umble, \& Murakami, 2006). Traditional DBR uses a three-buffer system to protect both the due-dates and detailed finite capacity schedule of the capacity constraint resources (CCR). This approach offers far more protection than merely keeping the CCR from starvation as a result of delay on the non-constraint resources. DBR assumes an internal CCR is active but in reality this is not always the case. In most cases, a company' s constraint is in the market which means even the CCR possesses sufficient protective capacity. Therefore, Schragenheim and Dettmer (2000), and Schragenheim, Weisenstern, and Schragenheim (2006) proposed a Simplified Drum-Buffer- Rope (SDBR) method. In this paper, we try to apply the SDBR concept into a schedule mechanism in a particular supply chain system, and use numerical analysis to test the efficiency of the proposed method.

\section{Basic Model and Nomenclature}

Before establishment of related model, we firstly setup a number of important parameters and assumptions inside the model as follows:

Q Quantity of customer product demand;

$q$ Quantity of the order sent by the distributor to the manufacturer for advance production before signing the formal contract with the customer;

$t$ The agreed termination time between the distributor and the customer, that is all the negotiations will be terminated at the $t$ moment regardless of whether the sales order is signed or not;

$\sigma$ The probability of signing the final contract between the distributor and customer at the $t$ moment;

$S$ The Setup time for activities such as procurement of raw materials and components, and equipment adjustment needed by the manufacturer for advance production according to the product 
quantity at $q$, thus $S_{\text {has actually become the decision variable of the distributor; }}$

$M$ The actual time put into advance production by the manufacturer at the $t$ moment in addition to the Setup time;

$V$ The production rate put into advance production by the manufacturer at the $t$ moment in accordance with capacity retention;

$\hat{v}$ The decided relative continuing production rate by the manufacturer at the $t$ moment in accordance with the signed sales order;

$u$ The product delivering rate by the distributor to customer through the VMI inventory system after the $t$ moment in accordance with the sales contract;

$p_{1}$ General market price per unit product, in this paper we assume it is the ex-factory price per unit product;

$p_{2}$ Product selling price signed by the distributor with the customer through the advance stock strategy based on the TOC;

$P$ The late delivery penalty per unit time promised in the contract signed between the distributor and the customer;

$\eta$ The supply chain coordination and decision risk coefficient borne by the manufacturer, that is, when there is a late delivery penalty to be paid to customer for late delivery arising from certain reasons, the $\eta$ proportion to be borne by the manufacturer, and the distributor will bear the left $1-\eta$ correspondingly;

C The cost coefficient for production of unit product by the manufacturer based on the Cobb-Douglas production function;

TPM Throughput of Manufacturer, or we can regard it as the manufacturer expected profit;

TCM Total Cost of Manufacturer, among which including all the variable costs during the production process;

TPD Throughput of Distributer, that is the distributor expected profit.

In addition, as in the practical environment, the raw materials or components purchased by the manufacturer during the Setup time can be used for production of other orders, and the adjusted equipment can also be applied into the other orders. Therefore, in the research of the article we will assume that if the order is canceled during the Setup time, the manufacturer will also cancel the production plan relatively without any additional cost. 
Based on the above settings, the distributor inside the established supply chain coordination model in accordance with the TOC will be the final link of the supply chain and facing directly with the supply chain system limits assumed by the SDBR mechanism inside the TOC-Market. Therefore, the market link has become the actual "bottleneck" of the complete supply chain. According to the principles of the TOC, a buffer inventory needs to be established before this link corresponding to ensure effective output of the market link, namely, the effective output of the whole supply chain. According to the aforementioned analysis, the buffer inventory level is related to the advance production, namely depending on the coordination strategy of the overall supply chain. Meanwhile in the coordination process of supply chain, advance production in the early process is actually the production process of stocking with reserved capacity in advance. Therefore, it is applying the SDBR mechanism inside the TOC to make corresponding control of the coordination for the whole supply chain under that strategy, with specific control mechanism

shown in Figure 2

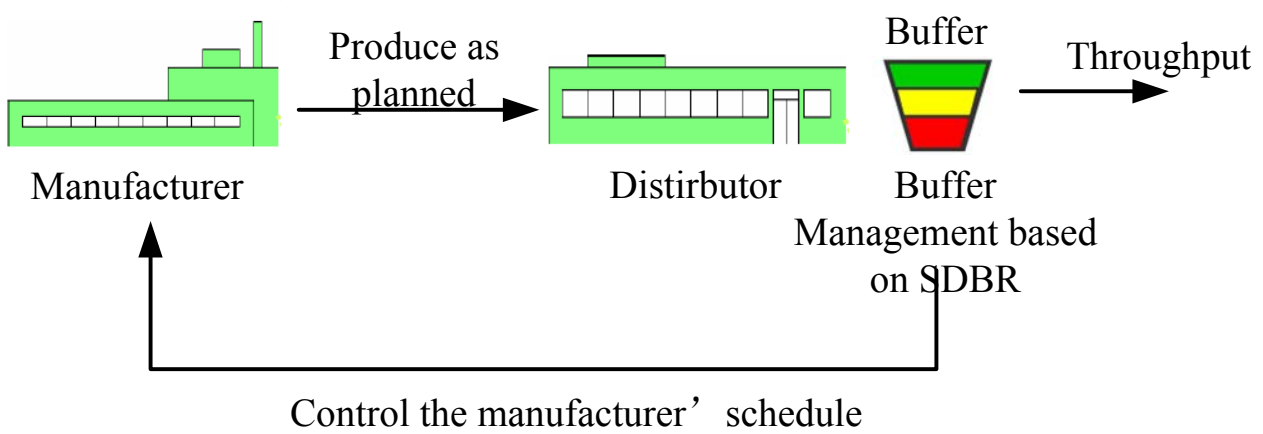

Figure 2 Supply chain coordination system based on SDBR

In the supply chain coordination system based on the SDBE, the supply chain buffer management strategy not only controls the decisions of the manufacturer's production plan and control, but also determines the effective outputs of the overall supply chain. Therefore, how to set up the specific buffer management is the core problem of this system.

Based on the above analysis, we have established a typical single-cycle supply chain system model inside the short life cycle product supply chain, in which the distributor faces a direct order of the market demand. At the time of 0 , the distributor obtained a needs intention of a certain customer with the specific demand as $Q$. Under general conditions, the customer and the distributor would carry out negotiations and related field 
research on the various details over the specific issues of sales and after sales, and meanwhile finalize the signing of the formal sales order contract at the $t$ moment based on the previous demand intentions. Of course, there was also relative possibility that the customer canceled the signing of the order at the $t$ moment due to some objective factors.

We can set $t$ as the continuous random variable, whose probability density function is $f(\cdot)$, and the probability distribution function is $F(\cdot)$. Assume the probability of the final signing of the distributor's sales order is $\sigma$, and then the probability of order missing is $1-\sigma$. After the distributor understands the customer's basic quantity needs at the 0 moment, and then a advance production order with the quantity at $q$ will be sent out to the manufacturer immediately, the released order without losing generality also occurs at the 0 moment, however, the significant decision need to be made by the distributor is the size of the variable $q$.

After the manufacturer received the advance production order from the distributor at the 0 moment, it would need a Setup time $S$ to go on with procurement of raw materials, components, and the equipment adjustment. The $\mathrm{S}$ was actually closely related with the distributor decision variable $q$ and became the actual decision variable. The actual production time put into production by the manufacturer was $M=t-S$, and then $M$ is also a continuous random variable, thus setting its probability density function as $f_{S}(\cdot)$, and its probability distribution function as $F_{S}(\cdot)$. Then we get the following equation from the definition of $M$ :

$$
F_{S}(M)=\operatorname{prob}\{t-S \leq M \mid t>S\}=\frac{F(M+S)-F(S)}{\bar{F}(S)}
$$

Among which the $\bar{F}(S)=1-F(S)$, and 0 - $t$ time period is actually based on the yellow area of the buffer management inside the SDBR supply chain system. The manufacturer starts production in accordance with certain capacity retention.

The red area of the buffering management is the time period from the $t$ moment of order signed to the promised delivery time of all the products, during which the 
manufacturer must put into corresponding capacity according to the delivery schedule to deliver on time. The relative parameter settings inside the buffering management are shown in Figure 3

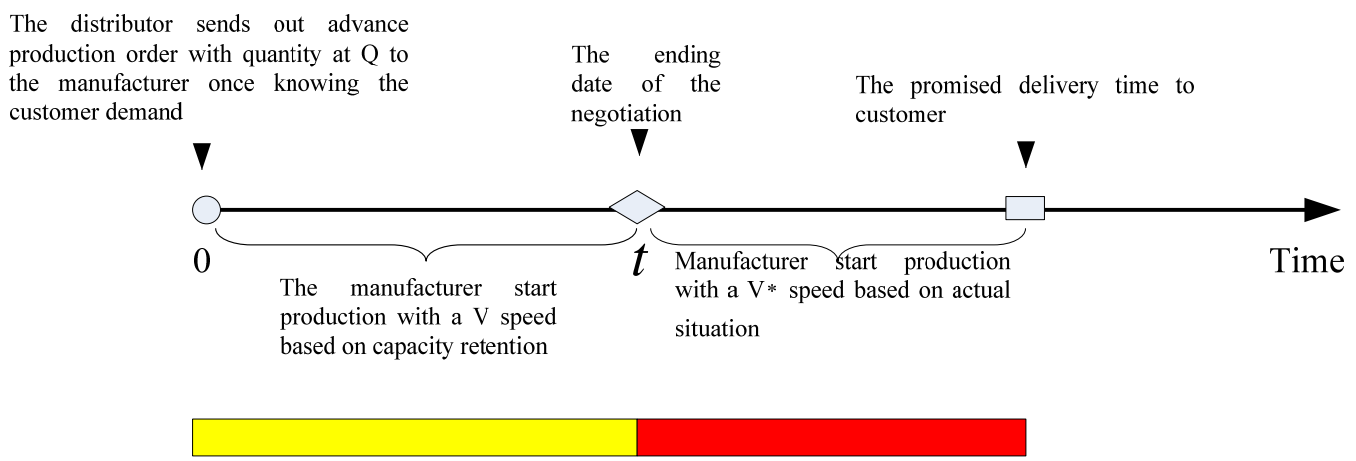

Figure3 SDBR system based buffer management

Here we set the manufacturer's production rate under certain capacity retention to be $v$, namely the time spend on each unit product is $\frac{1}{v}$. As inputs and outputs follow a power function relationship in the Cobb-Douglas production function, so we can set manufacturer's unit production cost under the general capacity as $c \cdot v^{\theta[160]}$.

At the $t$ moment, if the customer signs the sales order, and then the manufacturer will deliver the products already manufactured with a quantity at $q$ to the distributor. After the establishment of finished product inventory for the needed products of the corresponding customer, the distributor will build relative VMI replenishment system according to the sales order signed with the customer, and ensure the delivery of the needed $Q$ pieces of products to customer at a certain speed of $u$ within the specified delivery time, namely the average delivery time of each unit product is $\frac{1}{u}$. According to the relative strategy settings inside the SDBR system, the manufacturer will generally try to shorten the manufacturing time of the remaining products in terms of increasing the production capacity (such as emergency overtime production, increase production bottleneck required resources etc) after the $t$ moment. Here we set the manufacturer's average production rate can reach $\hat{v}$ after the $t$ moment, and then without losing generality we have $\hat{v} \geq v$, and the relative unit product manufacturing cost is $c \cdot \hat{v}^{\theta}$, 
among which $\hat{v}$ is the manufacturer's decision variable.

Here we set the general sales price at $p_{1}$, while the final sales price negotiated with the customer after using the advance delivery strategy set by the SDBR system can reach $p_{2}$, and $p_{2}>p_{1}$.

Since there is great uncertainty in the supply chain of the fashion products, there are possibilities that the sales order negotiation fails between the customer and distributor, and the customer canceled all its needs. However, therein according to the supply chain coordination principles, distributor shall provide relevant compensation policy for the already manufactured products by the manufacturer. The specific compensation amount is directly connected with the quantity of already manufactured product. Let's set the unit product compensation amount at $\delta$, and then the relative amount that the distributor shall compensate to the manufacturer is $\delta \cdot \min \{M \cdot v, Q\}$.

If the distributor's VMI replenishment system has delivered the entire inventory of the $q$ pieces product that have been supplied by the supplier before the $t$ moment to the customer, while the manufacturer is still unable to deliver the remaining products, and then the distributor's delivery process will be delayed, and affect the final delivery of customer's orders. A late delivery penalty thereof will be paid to the customer according to the sales contract signed, and then the late delivery cost of the unit time for the entire supply chain is $P$. Actually, the delivery time of the remaining products is not only related with the manufacturer's decision $\tilde{v}$, but also relevant with the quantity $q$ of the advance production order sent to the manufacturer by the distributor. Therefore, the production delayed cost is shared between the manufacturer and distributor. Once there is late delivery occurs for reasons of certain decision mistakes or some special reasons, and need to pay customer relevant late delivery penalty, the manufacturer shall bear the $\eta(0<\eta<1)$ part, and the distributor shall take the $1-\eta$ part, and then $\eta$ is the significant variable needed to be negotiated by the manufacturer and distributor in the manufacture and sales coordination decision of that supply chain.

Without losing generality and for ease of calculation, this article does not consider the inventory cost within the supply chain and among distributors and customers in this 
chapter. For the lots of inventory costs due to potential asymmetric information and damages to the supply chain overall return caused thereof will be discussed in subsequent chapters.

\section{The optimal decision analysis}

To study the manufacture and sales coordination strategy of the fashion products' supply chain based on TOC, we firstly need to do a relative analysis on the optimal decisions of all the links inside the supply chain and find out the optimal method for supply chain coordination based on this. Therefore, this section will analyze the best decisions of the manufacturers and distributors inside the supply chain, and then introduce the analysis results into the subsequent simulation example analysis in order to finally get the optimal coordination method of the fashion products' supply chain based on the TOC.

\subsection{Optimal decision analysis of the manufacturer under the condition of sales orders signed}

Firstly we will analyze the optimal production decision of the manufacturer under the condition of the sales orders signed inside the supply chain system based on the SDBR. According to the aforementioned model, the manufacturer will decide the average production rate $\tilde{V}$ of the remaining products based on the specifications of the sales order after the $t$ moment that the specific sales order signed between the distributor and customer. In addition to the production cost, the manufacturer also needs to consider the late delivery cost resulting from the possible late delivery. Therefore the manufacturer's objective at this time is how to minimize its own cost.

As the product quantity $q$ deliverable to the distributor at the $t$ moment has been fixed according to the distributor's advance production order(before the $t$ moment, that quantity is a variable $M \cdot v$ ), therefore the manufacturer will mainly consider the cost function thereafter: 


$$
\operatorname{OTC}(\hat{v})=P \cdot\left[\frac{(Q-q)}{\tilde{v}}-\frac{q}{u}\right]^{+} \cdot \eta+(Q-q) \cdot c \cdot \hat{v}^{\theta}
$$

Let's set $C_{1}=P \cdot\left[\frac{(Q-q)}{\hat{v}}-\frac{q}{u}\right]^{+} \cdot \eta$ inside the equation (2), and then $C_{1}$ is the supply chain late delivery cost borne by the manufacturer for reasons of late delivery according to the supply chain coordination decision; at the mean time we set $C_{2}=(Q-q) \cdot c \cdot \hat{v}^{\theta}$, and then $C_{2}$ is the production cost of the remaining products needed by the distributor after the $t$ moment.

Accordingly, we can first get the following lemmas:

Lemma 1. After the $t$ moment, if $\frac{u(Q-q)}{q}<v$, the optimal decision for continuous production of the manufacturer is $\hat{v}^{*}=v$ 。

Proof: $\operatorname{Let} v^{-1}=\xi, \quad \hat{v}^{-1}=\hat{\xi}$. Then $\xi, \hat{\xi} \geq 0$ and $C_{2}=(Q-q) \cdot c \cdot \hat{\xi}^{-\theta}$.

For $\forall \xi \leq \hat{\xi}$, there is $C_{2}(\xi) \leq C_{2}(\hat{\xi})$ for all the time, and

$$
C_{2}^{\prime \prime}(\hat{\xi})=\theta(\theta+1)(Q-q) \cdot c \cdot \hat{\xi}^{-\theta-2}>0,
$$

Then obviously:

for $\forall \xi \geq \hat{\xi}, C_{2}$ is the decreasing convex function on $\xi$.

And based on the definition, if $\hat{v} \geq \frac{u(Q-q)}{q}$, there is $C_{1}=0$ for all the time.

In conclusion, we have: if $\frac{u(Q-q)}{q}<v$, if and only if $\hat{\xi}=\xi$,

OTC $(\hat{v})=C_{1}+C_{2}$ gets the minimal value, namely the manufacturer's optimal decision is $\hat{v}^{*}=v$, QED.

With the same principle, we can also get the lemma 2 correspondingly:

Lemma 2. After the $t$ moment, if $\frac{u(Q-q)}{q}>v$, and then: 
(1) If $v<\frac{u(Q-q)}{q}<\left(\frac{P \cdot \eta}{c \cdot \theta}\right)^{\frac{1}{\theta+1}}$, the optimal decision for continuous production of the manufacturer is:

$$
\hat{v}^{*}=\frac{u(Q-q)}{q},
$$

(2) If $v<\left(\frac{P \cdot \eta}{c \cdot \theta}\right)^{\frac{1}{\theta+1}}<\frac{u(Q-q)}{q}$, the optimal decision for continuous production of the manufacturer is:

$$
\hat{v}^{*}=\left(\frac{P \cdot \eta}{c \cdot \theta}\right)^{\frac{1}{\theta+1}}
$$

(3) If $\left(\frac{P \cdot \eta}{c \cdot \theta}\right)^{\frac{1}{\theta+1}}<v<\frac{u(Q-q)}{q}$, the optimal decision for continuous production of the manufacturer is:

$$
\hat{v}^{*}=v \text { 。 }
$$

Proof: $\operatorname{Let} v^{-1}=\xi, \quad \hat{v}^{-1}=\hat{\xi}$. Then $\xi, \hat{\xi} \geq 0$, and

$$
\operatorname{OTC}(\hat{\xi})=P \cdot\left[(Q-q) \hat{\xi}-\frac{q}{u}\right]^{+} \cdot \eta+(Q-q) \cdot c \cdot \hat{\xi}^{-\theta}
$$

$\because$ After the $t$ moment, $v<\frac{u(Q-q)}{q}$, and then $\frac{q}{u(Q-q)}<\xi$

$\therefore$ Firstly, perform second derivation on the equation (3) within the interval $\left[\frac{q}{u(Q-q)}, \xi\right]$, we can get:

$$
\operatorname{OTC}^{\prime \prime}(\hat{\xi})=\theta(\theta+1)(Q-q) \cdot c \cdot \hat{\xi}^{-\theta-2}
$$

From the basic settings of all parameters inside the model, obviously we have $O T C^{\prime \prime}(\hat{\xi})>0$ inside the equation ( 4$)$, and then based on this we can get that the equation (4.3) is the decreasing convex function on $\xi$ within the interval 


$$
\left[\frac{q}{u(Q-q)}, \xi\right]
$$

Based on the nature of this function, let's perform first derivation on the equation (4.3), and then we get:

$$
\operatorname{OTC}^{\prime}(\hat{\xi})=-\theta(Q-q) \cdot c \cdot \hat{\xi}^{-\theta-1}+P \cdot(Q-q) \cdot \eta
$$

Let equation $(5)$ to be zero. Then for the function $\operatorname{OTC}(\hat{\xi})$ within the interval $\left[\frac{q}{u(Q-q)}, \xi\right]$, we can get the extreme point:

$$
\begin{gathered}
\hat{\xi}^{\nabla}=\left(\frac{\theta \cdot c}{P \cdot \eta}\right)^{\frac{1}{\theta+1}} \\
{\left[\frac{q}{u(Q-q)}, \xi\right] \text { is the domain of the function } \operatorname{OTC}(\hat{\xi}) .}
\end{gathered}
$$

However, with the changes of the decision variables $q$ and $\xi$, the interval $\left[\frac{q}{u(Q-q)}, \xi\right]$ will also change accordingly and lead to different locations with the extreme point $\hat{\xi}^{\nabla}$ :

1) If $\left(\frac{\theta \cdot c}{P \cdot \eta}\right)^{\frac{1}{\theta+1}}<\frac{q}{u(Q-q)}<\xi$, namely $v<\frac{u(Q-q)}{q}<\left(\frac{P \cdot \eta}{c \cdot \theta}\right)^{\frac{1}{\theta+1}}$, the extreme point $\hat{\xi}^{\nabla}$ locates at the left side of the domain $\left[\frac{q}{u(Q-q)}, \xi\right]$, by this time, the function $\operatorname{OTC}(\hat{\xi})$ gets the minimum value at point $\hat{\xi}=\frac{q}{u(Q-q)}$ within the domain, namely $\hat{v}=\frac{u(Q-q)}{q}$

2) If $\frac{q}{u(Q-q)}<\left(\frac{\theta \cdot c}{P \cdot \eta}\right)^{\frac{1}{\theta+1}}<\xi$, namely $v<\left(\frac{P \cdot \eta}{c \cdot \theta}\right)^{\frac{1}{\theta+1}}<\frac{u(Q-q)}{q}$, the extreme point $\hat{\xi}^{\nabla}$ locates within the domain $\left[\frac{q}{u(Q-q)}, \xi\right]$, by this time, 


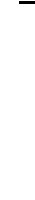

the function $\operatorname{OTC}(\hat{\xi})$ gets the minimum value at point $\hat{\xi}=\left(\frac{\theta \cdot c}{P \cdot \eta}\right)^{\frac{1}{\theta+1}}$ within the domain, namely $\hat{v}=\left(\frac{P \cdot \eta}{C \cdot \theta}\right)^{\frac{1}{\theta+1}}$

3) If $\frac{q}{u(Q-q)}<\xi<\left(\frac{\theta \cdot c}{P \cdot \eta}\right)^{\frac{1}{\theta+1}}$, namely $\left(\frac{P \cdot \eta}{c \cdot \theta}\right)^{\frac{1}{\theta+1}}<v<\frac{u(Q-q)}{q}$, the extreme point $\hat{\xi}^{\nabla}$ locates at the right side of the domain $\left[\frac{q}{u(Q-q)}, \xi\right]$,

by this time, the function $\operatorname{OTC}(\hat{\xi})$ gets the minimum value at point $\hat{\xi}=\xi$ within the domain, namely $\hat{v}=v$, QED.

In this chapter, in order to simplify the subsequent presentation, set $\omega=\max \left[\left(\frac{P \cdot \eta}{c \cdot \theta}\right)^{\frac{1}{\theta+1}}, v\right]$. According to the Lemma 1 and Lemma 2, we get the following theorem about the optimal decision of the manufacturer:

Theorem 1: If the end customer demand order is confirmed and signed by the distributor and customer at the $t$ moment and the advance production quantity already sent by the distributor to the manufacturer before the signing of the order is $q$, and then:

(1) When $\frac{u(Q-q)}{q}<v$, after the $t$ moment, the optimal decision of the manufacturer for production of the remaining products is $\hat{v}^{*}=v$, the distributor's delivery will not be delayed for products not being able to be supplied in time;

(2) When $v<\frac{u(Q-q)}{q}<\left(\frac{\eta \cdot P}{\theta \cdot c}\right)^{\frac{1}{\theta+1}}$, after the $t$ moment, the optimal decision of the manufacturer for production of the remaining products is $\hat{v}^{*}=\frac{u(Q-q)}{q}$, by this time, the distributor's delivery will not be delayed for products not being able to be supplied in time; 
(3) When $\omega<\frac{u(Q-q)}{q}$, after the $t$ moment, the optimal decision of the manufacturer for production of the remaining products is $\hat{v}^{*}=\omega$, but the distributor's delivery will be delayed resulting from the manufacturer being unable to supply the remaining products in time, the relative delayed time is $\frac{(Q-q)}{\omega} \cdot-\frac{q}{u}$

Proof: Can be comprehensively proved by the Lemma 1 and Lemma 2, proof omission.

In the subsequent simulation analysis, we need to consider not only the production cost of the manufacturer after the $t$ moment, but also the expected effective outputs of the manufacturer in the entire production process. According to Corollary 1 shows that manufacturer expected effective output function will need to consider the two different cases of $\left(\frac{P \cdot \eta}{C \cdot \theta}\right)^{\frac{1}{\theta+1}}<v$ and $v<\left(\frac{P \cdot \eta}{C \cdot \theta}\right)^{\frac{1}{\theta+1}}$, as well as the total production cost TCM of the entire process. At the same time, set the unit product loss of the manufacturer at $l$ before the sales order not signed by customer, and after the compensation made by the distributor to manufacturer accordingly. According to Corollary 1, the manufacturer expected effective output function will be calculated respectively according to the aforementioned two different situations, in order to simplify the subsequent presentation, set $\left(\frac{P \cdot \eta}{C \cdot \theta}\right)^{\frac{1}{\theta+1}}=\varpi$, and then there are:

(1)When $\varpi<v$, and then $\omega=v$, the corresponding expected effective output function is:

$$
\begin{aligned}
\operatorname{TPM}_{1}= & \sigma \cdot\left\{p_{1} \cdot Q-F(S) \cdot \frac{P \cdot Q}{v} \cdot \eta-\bar{F}(S) \cdot \eta \cdot P \cdot \int_{0}^{\frac{u Q}{v(u+v)}}\left[\frac{(Q-M \cdot v)}{v}-\frac{M \cdot v}{u}\right] d F_{t}(M)-Q \cdot c \cdot v^{\theta}\right\} \\
& -(1-\sigma) \cdot \bar{F}(S) \cdot l \cdot E[\min (M \cdot v, Q)]
\end{aligned}
$$

(2) When $\varpi>v$, and then $\omega=\varpi$, the corresponding expected effective output function is: 


$$
\begin{aligned}
T P M_{2}= & \sigma \cdot\left\{p_{1} \cdot Q-F(S) \cdot\left(\eta \frac{P \cdot Q}{\varpi}+c \cdot \varpi^{\theta} \cdot Q\right)-\bar{F}(S) \cdot\left\{T C M+\eta \cdot P \cdot \int_{0}^{\frac{u Q}{v(u+\sigma)}}\left[\frac{(Q-M \cdot v)}{\varpi}-\frac{M \cdot v}{u}\right] d F_{t}(M)\right\}\right. \\
& -(1-\sigma) \cdot \bar{F}(S) \cdot l \cdot E[\min (M \cdot v, Q)]
\end{aligned}
$$

there into:

$$
\begin{aligned}
T C M= & \int_{0}^{\frac{u Q}{v(u+\sigma)}}(Q-M \cdot v) \cdot c \cdot \varpi^{\theta} d F_{S}(M)+\int_{\frac{u Q}{v(u+\sigma)}}^{\frac{u Q}{v(u+v)}}(Q-M \cdot v) \cdot c \cdot\left(\frac{u Q}{M \cdot v}-1\right)^{\theta} d F_{S}(M) \\
& +\int_{\frac{u Q}{v(u+v)}}^{\frac{Q}{v}}(Q-M \cdot v) \cdot c \cdot v^{\theta} d F_{S}(M)+E[\min (M \cdot v, Q)] \cdot c \cdot v^{\theta}
\end{aligned}
$$

\subsection{Optimal decision analysis of the distributor}

In the SDBR based supply chain system, the main problem faced by distributor is what the quantity is to be sent to the manufacturer for advance production, namely how to decide the decision variable $q$. If the product quantity $q$ for advance production is too big, once the negotiation between the distributor and customer for signing the sales order is unsuccessful at the $t$ moment, and the order is not signed, and then the compensation amount $\delta \cdot \min \{M \cdot v, Q\}$ that the distributor needs to pay to the manufacturer for advance production is relatively larger, thus brings distributor the risk of order losing. Conversely, if the $q$ is too small, after sales order signed at the $t$ moment, the quantity of products already produced by manufacturer and available for deliver may be small, and then resulting delays in the distributor's delivery process for reasons of the upstream products delivery being unable to follow-up timely, thus the distributor will face the risk of late delivery penalty from the customer. On the other hand, the sales order may be canceled before the Setup time $\mathrm{S}$, that is $t<S$. Based on the foregoing assumptions, distributor will not have any costs in this case.

In the process of specific simulation analysis, when calculating the compensation for product manufactured by the distributor to the manufacturer under the condition of the lost sales order, if compensation per unit product is $\delta$ based on the aforementioned, the total compensation amount is $\delta \cdot \min \{M \cdot v, Q\}$, and then the overall calculation process in the specific calculation will be very complex, and therefore without loss of 
generality, for the setting of the distributor desired output function, namely distributor desired profit function in this paper, we adopt the time-related orders loss compensation method proposed by Huang in its literature ${ }^{[161]}$, that is taking into account of the product quantity already manufactured within the $t$ time and management costs and other factors, considering the actual production time $M$ is proportional in the linear function. Define the compensation cost for unit production time as $\gamma$, and then the total compensation expense to be paid by the distributor is $\gamma \cdot M$, with the maximizing the distributor's expected profit as the decision-making objective under the condition of uncertain orders. Neglect the distributor's inventory, management and other costs inside the objective function, and then the distributor's objective function is:

$$
\begin{aligned}
\operatorname{TPD}(S)= & \sigma \cdot\left\{\left(p_{2}-p_{1}\right) \cdot Q-F(S) \cdot\left[(1-\eta) \cdot P \cdot \frac{Q}{v}\right]-\bar{F}(S) \cdot\left\{(1-\eta) \cdot P \cdot E\left[\frac{(Q-M \cdot v)^{+}}{\hat{v}}-\frac{\min (M \cdot v, Q)}{u}\right]^{+}\right\}\right\} \\
& -(1-\sigma) \cdot \bar{F}(S) \cdot \gamma \cdot E[M]
\end{aligned}
$$

According to Corollary 1 , when $\omega<\frac{u(Q-q)}{q}$, after the $t$ moment, the manufacturer's optimal decision for production of the remaining products is $\hat{v}=\omega$, by this time distributor's delivery will be delayed resulting from the manufacturer's being unable to supply the remaining products in time, and then based on this we can simplify partials of the equation (7), and:

$$
\begin{aligned}
E\left[\frac{(Q-M \cdot v)^{+}}{\hat{v}}-\frac{\min (M v, Q)}{u}\right]^{+} & =\frac{v(\omega+u)}{\omega \cdot u \cdot \bar{F}(S)} \cdot\left[\int_{S}^{\frac{u \cdot Q}{v(\omega+u)}+S} F(t) d t-\frac{u \cdot Q}{v(\omega+u)} \cdot F(S)\right] \\
E[M] & =\frac{1}{\bar{F}(S)} \cdot \int_{S}^{\infty} \bar{F}(t) d t
\end{aligned}
$$

According to (8) (9), the equation (7) can be simplified as:

$$
\begin{aligned}
\operatorname{TPD}(S)= & \sigma \cdot\left\{\left(p_{2}-p_{1}\right) \cdot Q-F(S) \cdot\left[(1-\eta) \cdot P \cdot \frac{Q}{\omega}\right]-\left\{(1-\eta) \cdot P \cdot \frac{v(u+\omega)}{u \cdot \omega}\left[\int_{s}^{\frac{u Q}{v(\omega+u)}+S} F(t) d t-\frac{u Q}{v(\omega+u)} \cdot F(S)\right]\right\}\right\} \\
& -(1-\sigma) \cdot \gamma \cdot \int_{s}^{\infty} \bar{F}(t) d t
\end{aligned}
$$


First derivation to the equation (10), and then get:

$$
\begin{aligned}
\operatorname{TPD}^{\prime}(S) & =-\sigma \cdot(1-\eta) \cdot P \cdot \frac{v(u+\omega)}{u \cdot \omega} \cdot\left[F\left(\frac{u Q}{v(u+\omega)}+S\right)-F(S)\right]+(1-\sigma) \cdot \gamma \cdot \bar{F}(S) \\
& =-\bar{F}(S) \cdot\left\{\sigma \cdot(1-\eta) \cdot P \cdot \frac{v(u+\omega)}{u \cdot \omega} \cdot F_{S}\left(\frac{u Q}{v(u+\omega)}\right)-\gamma \cdot(1-\sigma)\right\}
\end{aligned}
$$

When equation (11) is set as zero, and then get:

$$
F_{S}\left(\frac{u Q}{v(u+\omega)}\right)=\frac{u \cdot \omega \cdot \gamma \cdot(1-\sigma)}{\sigma \cdot(1-\eta) \cdot P \cdot v(u+\omega)}
$$

Set $S=S^{\nabla}$, that is $\operatorname{TP} D^{\prime}\left(S^{\nabla}\right)=0$, meanwhile set the Setup time needed by the manufacturer for production of all the $Q$ pieces as $S^{Q}$, and then we can get the Theorem 2 corresponding with the optimal decision of the distributor:

Theorem 2: Define the distributor sends out advance production order with quantity at $q$ to the manufacturer at the 0 moment, the relative Setup time needed by the manufacturer is:

$$
\hat{S}= \begin{cases}\infty & \lim _{S \rightarrow \infty} T P D^{\prime}(S) \geq 0 \\ S^{Q} & T P D^{\prime}(S)<0 \\ S^{\nabla} & \text { ot her } s\end{cases}
$$

(1) If the distributor and customer sign the order within the Setup time $\hat{S}$ of the manufacturer, after order signed, the distributor will immediately send the remaining production order $Q-q$ to the manufacturer and continuously deliver the products to customer through the VMI replenish system after the delivery of manufacturer.

(2) If the customer cancels the order within the Setup time $\hat{S}$ of the manufacturer, the distributor will immediately cancel the corresponding advance production order.

(3) For other general cases, the distributor sends out advance production order with quantity at $q$ to the manufacturer, the relative Setup time is $\hat{S}$, after the $t$ moment, sends out production order of the remaining quantity $Q-q$ to the manufacturer. 
Proof: $\operatorname{Set} \frac{u \cdot \omega \cdot \gamma \cdot(1-\sigma)}{\sigma \cdot(1-\eta) \cdot P \cdot v(u+\omega)}=\tau$

by $T P D^{\prime}\left(S^{\nabla}\right)=0$, we get $F_{S^{\nabla}}\left(\frac{u Q}{v(u+\omega)}\right)=\tau$

If set the probability distribution function $F(\cdot)$ of time $t$ as product life distribution function inside the reliability theory. Then the corresponding $F_{S}(\cdot)$ is the conditional failure probability distribution. According to its definition, for a product with a life period of $S$, the $F_{S}(M)$ can represent the specific failure probability within the $M$ time after the $S$ moment.

Since in the environment discussed inside the article, within the actual production time $M$ after the $S$ moment, regardless of whether the order can be signed or not, the probability to conclude the negotiations between the distributor and customer is gradually increasing with the increase of the Setup time $S$. Therefore, we can regard the $t$ time has the nature of increasing failure rate (IFR). Then based on the IFR nature of the $t$, hence get:

For $\forall M>0, F_{S}(M)$ is the monotone increasing function with respect to $M$, and then $\operatorname{TPD}(S)$ is quasi concave function, its image form is a single-peak curve. Based on this, we get:

1 ) If $\lim _{S \rightarrow \infty} F_{S}\left(\frac{u Q}{v(u+\omega)}\right) \leq 1 \leq \tau, \lim _{S \rightarrow \infty} T P D^{\prime}(S) \geq 0$, the $T P D(S)$ is monotone increasing, and then at this time, the distributor's optimal decision is to send the production order with full quantity $Q$ to the manufacturer after signing sales order with customer.

2) If $F_{S}\left(\frac{u Q}{v(u+\omega)}\right)>\tau, T P D^{\prime}(S)<0$, the $T P D(S)$ is monotone increasing, and then at this time, the distributor's optimal decision is to send the advance production order with quantity $Q$ to the manufacturer immediately after knowing the customer needed product quantity $Q$ at the 0 moment.

3 ) Under general conditions, the $\operatorname{TPD}(S)$ get the maximum value at the point of $S=S^{\nabla}$, QED.

In addition, in the entire supply chain coordination process, the undertaken 
coefficient $\eta$ needs to be firstly determined through consultations between the manufacturer and distributor. Inside the fashion products supply chain studied in this paper, Corollary 1 and Corollary 2 are in fact based on the appropriate optimal decisions made to maximize interests of the various aspects in the supply chain. However, the actual confirmation must make the two parties of manufacturing and selling obtain the expected profits, otherwise, the whole supply chain is very difficult to achieve coordination, namely the necessary condition to confirm $\eta$ is first meetTPM,TPD $>0$. Therefore, in this chapter we will use the corresponding simulation cases based on the parameters set to research the $\eta$ values and the corresponding correlation among parameters

\section{Analysis of Simulation Calculation}

In simulation calculation setting of the supply chain coordination strategy, firstly, since the Gamma distribution has the IFR nature, therefore we assume that the final order signing time $t$ of the distributor and customer obey the Gamma distribution $(6,6)$ in the simulation system. After the $t$ moment, according to the coordination strategy confirmed by the manufacturer and distributor, if manufacturer fails to supply the remaining undelivered products and need to pay the late delivery penalty specified in the relevant agreement signed with customer, the manufacturer shall undertake the $\eta(0<\eta<1)$ part, and then distributor shall bear the corresponding $1-\eta$ part. Firstly confirm the other important parameters in the simulation system, the specific settings are: $C=1, \quad \theta=2, S=300, W=60, R=600, l_{s}=0.2, l_{m}=0.5, \quad s=5$ 。

(1) Firstly through simulation calculation, we will analyze the influence of supply chain coordination parameter $\eta$ to the expected effective outputs of the manufacturer and distributor, and the possible influence of the manufacturing and selling parties' supply chain coordinated strategy to the late delivery time of the distributor. In the simulation calculation of this part, we first set $\eta=0.1 \sim 0.9, \sigma=0.7, P=2000, Q=100$ to carry out relative simulation calculations. The results are shown in Table 1

Table 1 The expected profits of the manufacturing and selling parties in the supply chain

\begin{tabular}{|l|l|l|l|l|l|l|l|l|l|}
\hline$\eta$ & 0.1 & 0.2 & 0.3 & 0.4 & 0.5 & 0.6 & 0.7 & 0.8 & 0.9 \\
\hline
\end{tabular}




\begin{tabular}{|c|c|c|c|c|c|c|c|c|c|}
\hline \hline $\begin{array}{c}\text { Optimal decision of } \\
\text { distributor } \hat{S}\end{array}$ & 9.7 & 11.4 & 13.1 & 15 & 17.2 & 20.2 & 25.4 & 38 & $\infty$ \\
\hline $\begin{array}{c}\text { Effective output of the } \\
\text { manufacturer (TPM) }\end{array}$ & 2259 & 2132 & 1917 & 1575 & 1001 & -58 & -2344 & -8403 & -15376 \\
\hline $\begin{array}{c}\text { Effective output of the } \\
\text { distributor (TPD) }\end{array}$ & 15702 & 15931 & 16161 & 16383 & 16630 & 16939 & 17383 & 18153 & 19550 \\
\hline $\begin{array}{c}\text { Expected late delivery } \\
\text { time }\end{array}$ & 0.3221 & 0.3442 & 0.3725 & 0.4169 & 0.4854 & 0.5926 & 0.7797 & 1.1675 & 10.3574 \\
\hline
\end{tabular}

From the Table 1, we can find that with the increasing of $\eta$, the distributor is actually transferring the late delivery cost borne by itself to the manufacturer, which therefore leading to the decrease of the manufacturer's expected output and increase of the distributor's expected output. Although the specific value of the overall coordination parameter $\eta$ is determined through negotiation by the distributor and manufacturer, yet no matter whether the distributor has a strong positive position inside the supply chain or not, the value of $\eta$ still must firstly ensure the manufacturer can obtain appropriate profit rate from the whole coordination process. Therefore in the calculation case, the maximum value of the $\eta$ can only be 0.5 , that is once there is possible later delivery and the corresponding penalty, the two parties of the supply chain shall bear at least half of the late delivery penalty, otherwise the manufacturer's expected profit will be negative, and then the manufacturer will not have any initiative in participation of the overall supply chain coordination process.

It is also worth noting that, although with the increase of the $\eta$, the distributor gradually transferred the overall late delivery costs and risks to the manufacturer, yet to a certain extent, this can cause manufacturer to increase production capacity for the remaining products of the second phase after signing of the sales order. However, a more noteworthy thing is that this behavior can't shorten the expected late delivery time of the whole supply chain to the end customers. The main reason for this phenomenon is that: as the late delivery cost borne by distributor decreases, the distributor's decision variable $q$ will gradually increase, thus brings the manufacturer's Setup time $\hat{S}$ increase gradually, thereby greatly increasing the late delivery probability of the overall supply chain. This also indicates from another aspect that the influence of the distributor's direct 
decision variable $q$ to the late delivery time is larger than the influence of the manufacturer's direct decision variable $\hat{v}$ to the expected late delivery time. In short, inside the short life cycle product supply chain studied in this chapter, the finally promised delivery time to customer is actually completely determined by the distributor, meanwhile, the distributor' $\mathrm{s}$ advance production decision will directly affect whether all the products of the required quantity can be delivered to customer within the promised delivery time at last.

(2) Here, based on the different parameter settings of another group, we will research the influences of the distributor's sales order signing probability on the manufacturer's expected effective output, the distributor's expected effective output and coordination of policies of the overall supply chain. In the data setting of that group's simulation calculation, we will set the distributor's sales order signing probability with customer at the $t$ moment as $\sigma$ with ranges at $0.1 \sim 0.9$, meanwhile set the late delivery penalty cost of the unit time as $P=2000$, and the total customer demanded product quantity as $Q=100$, and then use them as basic inputs to carry out simulation calculation. The final results are shown in Table 2 and Table 3.

Table 2 Manufacturer's expected effective outputs under different order signing probabilities

\begin{tabular}{|c|c|c|c|c|c|c|c|c|c|}
\hline $\begin{array}{c}\text { र } \\
\sigma\end{array}$ & 0.1 & 0.2 & 0.3 & 0.4 & 0.5 & 0.6 & 0.7 & 0.8 & 0.9 \\
\hline 0.1 & -50 & -426 & -744 & -1029 & -1290 & -1534 & -1765 & -1985 & -2197 \\
\hline 0.2 & -16 & -849 & -1489 & -2057 & -2580 & -3068 & -3530 & -3971 & -4393 \\
\hline 0.3 & 478 & -334 & -1667 & -3034 & -3870 & -4602 & -5295 & -5956 & -6590 \\
\hline 0.4 & 955 & 449 & -483 & -2018 & -4263 & -6126 & -7060 & -7941 & -8786 \\
\hline 0.5 & 1403 & 1080 & 499 & -482 & -2190 & -5170 & -8698 & -9927 & -10983 \\
\hline 0.6 & 1835 & 1629 & 1268 & 676 & -348 & -2287 & -6290 & -11843 & -13179 \\
\hline 0.7 & 2259 & 2132 & 1917 & 1575 & 1001 & -58 & -2344 & -8403 & -15376 \\
\hline 0.8 & 2677 & 2608 & 2492 & 2316 & 2030 & 1523 & 475 & -2376 & -14548 \\
\hline 0.9 & 3091 & 3063 & 3018 & 2951 & 2847 & 2672 & 2333 & 1486 & -2390 \\
\hline
\end{tabular}

Table3 Distributor's expected effective outputs under different order signing probabilities

\begin{tabular}{|c|c|c|c|c|c|c|c|c|c|}
\hline \multicolumn{1}{|c|}{$\mid x$} & 0.1 & 0.2 & 0.3 & 0.4 & 0.5 & 0.6 & 0.7 & 0.8 & 0.9 \\
\hline 0.1 & -600 & 264 & 909 & 1371 & 1740 & 2051 & 2324 & 2569 & 2793 \\
\hline
\end{tabular}




\begin{tabular}{|c|c|c|c|c|c|c|c|c|c|}
\hline 0.2 & -1110 & 528 & 1817 & 2743 & 3480 & 4103 & 4649 & 5138 & 5586 \\
\hline 0.3 & 316 & 1514 & 2846 & 4116 & 5220 & 6154 & 6973 & 7707 & 8379 \\
\hline 0.4 & 3380 & 4157 & 5024 & 5952 & 7026 & 8206 & 9297 & 10276 & 11171 \\
\hline 0.5 & 7152 & 7675 & 8239 & 8827 & 9525 & 10435 & 11623 & 12846 & 13964 \\
\hline 0.6 & 11304 & 11657 & 12024 & 12393 & 12818 & 13369 & 14177 & 15415 & 16757 \\
\hline 0.7 & 15702 & 15931 & 16161 & 16383 & 16630 & 16939 & 17383 & 18153 & 19550 \\
\hline 0.8 & 20286 & 20419 & 20549 & 20668 & 20796 & 20950 & 21161 & 21513 & 22367 \\
\hline 0.9 & 25035 & 25093 & 25147 & 25194 & 25242 & 25297 & 25368 & 25478 & 25725 \\
\hline
\end{tabular}

Based on the simulation calculation results, we can get from the Table 2 that if the distributor's sales order signing probability $\sigma$ at the $t$ moment is very small, even if the proportion $\eta$ of the late delivery cost borne by the manufacturer is very small, it is still very difficult for the manufacturer to obtain the real effective output. While with the increase of the final order signing probability $\sigma$, the manufacturer's expected effective output will also increase accordingly.

On the other hand, we can also draw from Table 3 that along with $\sigma$ value increase of the sales order final signing probability, the influence of value changes in supply chain coordination strategy $\eta$ to the distributor's expected effective output will become smaller and smaller. The most obvious is that when the order final signing probability reaches $\sigma=0.9$, within the domain of supply chain coordination strategy $\eta$,the distributor's expected effective output is generally stable regardless of how the $\eta$ value changes.

Therefore, based on the simulation calculation and analysis over the manufacturer's expected effective output, the distributor's expected effective output and the supply chain overall coordination strategy under the different order signing probabilities, we get the relative conclusion, namely: under the condition that final sales order signing probability is larger, during supply chain coordination decision process, the distributor shall be more active and make some decision adjustments in favor of increasing the manufacturer's expected output when negotiating and confirming the value of $\eta$ with manufacturer to make the manufacturer get more profits, so that it can have a greater positive participation to the overall coordination of the supply chain process, thus further increasing the effective output of the overall supply chain. 
(3) Based on the same system framework, we will further study the relevant impacts of the unit time delay cost $P$ to the manufacturer expected effective output, the distributor's expected effective output and the supply chain overall coordination strategy under different value conditions. Therefore, in the simulation calculation setting of this section, we will set values of the unit time delay cost $P$ separately as 1000,1600,2500, meanwhile set the order signing probability as $\sigma=0.7$, the total customer demanded product quantity as $Q=100$, and then use them as basic inputs to carry out simulation calculation. The final results are shown in Table 4, 5.

According to the results of simulation calculation, we can conclude from Table 5 that the values of late delivery unit time penalty cost $P$ have little effect on the distributor's expected effective output, and the value of distributor's expected effective output TPM is generally in a relatively stable level, regardless of how value range changes of the $P$. However the results that we get in Table 4.4 is that when the $P$ value is relatively small, the manufacturer expected effective output is more sensitive to the size of supply chain coordination policy value $\eta$. This is because when the $P$ is smaller, as the increase of $\eta$, the distributor borne late delivery cost ratio $1-\eta$ is gradually decreasing, thus making the distributor's direct decision variable $q$ become larger and larger, and further leading to the increase of manufacturer's Setup time $\hat{S}$. While the increasing value of the $\hat{S}$ means that manufacturer needs to take greater proportion of late delivery cost on one hand, and also need to maximize the production capacity for remaining products of the second phase after signing of the sales order.

Therefore, based on the simulation calculation analysis of the different unit time delay cost $P$, we can get the relative conclusion: when the unit time delay cost $P$ is relatively small, during supply chain coordination decision process, the manufacturer shall be more active and make some decision adjustments in favor of increasing its expected output when negotiating and confirming the value of $\eta$ with distributor, that is trying to minimize the value of $\eta$ in the supply chain coordination strategy.

(1) Based on the same system framework, we will further study the relevant impacts of the customer product demand quantity $Q$ to the manufacturer expected effective output, the distributor's expected effective output and the supply chain overall coordination strategy under different value conditions. Therefore, in the simulation 
calculation parameter input settings of this section, we will set values of customer product demand quantity $Q$ separately as 50,150,350, meanwhile set the order signing probability as $\sigma=0.7$, the unit time late delivery cost as $P=2000$ to carry out simulation. The final results are shown in Table 6,7 .

According to the results of simulation calculation, the results that we get from Table 6, 7 are quite different with what we get from Table 4, 5. When the total customer product demand $Q$ is relatively small, both the manufacturer's expected effective output and distributor's expected effective output is quite sensitive to the $\eta$ value ranges. While this also means that in the supply chain coordination process, the manufacturer and distributor will meet with difficulties when negotiating and confirming the $\eta$ value of the coordination strategy, the stronger party of the supply chain will have more voice right. However, with obvious contrast, under the condition of a larger $Q$ value of the total customer product demand quantity, the manufacturer's expected effective output TPM is significantly more sensitive than the distributor expected effective output TPM to the $\eta$ values of the supply chain coordination strategy. Therefore under this condition, the distributor can assume more proportion $1-\eta$ of the late delivery cost.

Therefore, based on the simulation calculation analysis of the different customer product demand $Q$, we can get the relative conclusion: When distributor finds potential customers in the market, the customers with larger product demand quantities should be the first contacting objectives, in order to avoid the obstacles and executing difficulties caused by the two parties' sensitivity to the supply chain coordination strategy variable $\eta$ when applying the TOC based supply chain coordination strategy. While under the condition that the total customer product demand quantity is larger, during supply chain coordination decision process, the distributor shall actively bear more late delivery cost proportion when negotiating and confirming the value of $\eta$ with manufacturer to make it have a greater positive participation to the overall coordination of the supply chain. 
Table 4 Manufacturer's expected effective output under different late delivery costs

\begin{tabular}{|c|c|c|c|c|c|c|c|c|c|c|c|c|c|c|c|c|c|c|}
\hline \multirow[b]{2}{*}{$P$} & \multicolumn{2}{|c|}{0.1} & \multicolumn{2}{|c|}{0.2} & \multicolumn{2}{|c|}{0.3} & \multicolumn{2}{|c|}{0.4} & \multicolumn{2}{|c|}{0.5} & \multicolumn{2}{|c|}{0.6} & \multicolumn{2}{|c|}{0.7} & \multicolumn{2}{|c|}{0.8} & \multicolumn{2}{|c|}{0.9} \\
\hline & TPM & $\hat{s}$ & TPM & $\hat{s}$ & TPM & $\hat{s}$ & TPM & $\hat{s}$ & TPM & $\hat{s}$ & TPM & $\hat{s}$ & TPM & $\hat{s}$ & TPM & $\hat{s}$ & TPM & $\hat{s}$ \\
\hline 1000 & 2246 & 13.6 & 2125 & 14.5 & 1905 & 16.2 & 1484 & 18.9 & 740 & 22.4 & -691 & 27.9 & -3662 & 39.5 & -7195 & 100.8 & -8132 & $\infty$ \\
\hline 1600 & 2255 & 10.8 & 2137 & 12 & 1911 & 14 & 1547 & 16 & 926 & 18.6 & -238 & 22.2 & -2768 & 28.5 & -8923 & 46.4 & -12670 & $\infty$ \\
\hline 2500 & 2262 & 8.8 & 2129 & 10.8 & 1923 & 12.3 & 1602 & 14 & 1070 & 16 & 100 & 18.7 & -1964 & 22.9 & -7595 & 32.5 & -18515 & 132.2 \\
\hline
\end{tabular}

Table 5 Distributor's expected effective output under different late delivery costs

\begin{tabular}{|c|c|c|c|c|c|c|c|c|c|c|c|c|c|c|c|c|c|c|}
\hline \multirow[b]{3}{*}{$P$} & \multicolumn{2}{|c|}{0.1} & \multicolumn{2}{|c|}{0.2} & \multicolumn{2}{|c|}{0.3} & \multicolumn{2}{|c|}{0.4} & \multicolumn{2}{|c|}{0.5} & \multicolumn{2}{|c|}{0.6} & \multicolumn{2}{|c|}{0.7} & \multicolumn{2}{|c|}{0.8} & \multicolumn{2}{|c|}{0.9} \\
\hline & & & & & & & & & & & & & & & & & & \\
\hline & $T P D$ & $\hat{s}$ & $T P D$ & $\hat{s}$ & $T P D$ & $\hat{s}$ & $T P D$ & $\hat{s}$ & $T P D$ & $\hat{s}$ & $T P D$ & $\hat{s}$ & $T P D$ & $\hat{s}$ & $T P D$ & $\hat{s}$ & $T P D$ & $\hat{s}$ \\
\hline 1000 & 16164 & 13.6 & 16260 & 14.5 & 16457 & 16. 2 & 16746 & 18.9 & 17079 & 22.4 & 17512 & 27.9 & 18149 & 39.5 & 19100 & 100.8 & 20087 & $\infty$ \\
\hline 1600 & 15834 & 10.8 & 15999 & 12 & 16245 & 14 & 16486 & 16 & 16756 & 18.6 & 17098 & 22.2 & 17596 & 28.5 & 18451 & 46.4 & 19750 & $\infty$ \\
\hline 2500 & 15582 & 8.8 & 15869 & 10.8 & 16084 & 12.3 & 16290 & 14 & 16517 & 16 & 16799 & 18.7 & 17197 & 22.9 & 17885 & 32.5 & 19317 & 132.2 \\
\hline
\end{tabular}


Table6 Manufacturer's expected effective output under different demand quantities

\begin{tabular}{|c|c|c|c|c|c|c|c|c|c|c|c|c|c|c|c|c|c|c|}
\hline & \multicolumn{2}{|c|}{0.1} & \multicolumn{2}{|c|}{0.2} & \multicolumn{2}{|c|}{0.3} & \multicolumn{2}{|c|}{0.4} & \multicolumn{2}{|c|}{0.5} & \multicolumn{2}{|c|}{0.6} & \multicolumn{2}{|c|}{0.7} & \multicolumn{2}{|c|}{0.8} & \multicolumn{2}{|c|}{0.9} \\
\hline$Q \backslash$ & $T P M$ & $\hat{s}$ & $T P M$ & $\hat{s}$ & $T P M$ & $\hat{S}$ & $T P M$ & $\hat{s}$ & TPM & $\hat{S}$ & TPM & $\hat{s}$ & $T P M$ & $\hat{S}$ & TPM & $\hat{S}$ & TPM & $\hat{s}$ \\
\hline 50 & 1076 & 14.9 & 910 & 17.1 & 617 & 19.7 & 131 & 22.7 & -709 & 26.9 & -2250 & 33.6 & -4968 & 48.7 & -6949 & 145.6 & -27688 & $\infty$ \\
\hline 150 & 3438 & 6.4 & 3317 & 8.1 & 3115 & 9.7 & 2806 & 11.3 & 2309 & 13.1 & 1425 & 15.4 & -422 & 18.9 & -5559 & 26.1 & -22762 & 74.3 \\
\hline 350 & 8058 & 0 & 8066 & 0 & 7743 & 0.08 & 7311 & 2 & 6698 & 3.8 & 5761 & 5.7 & 4112 & 8.1 & 286 & 12 & -18078 & 23.6 \\
\hline
\end{tabular}

Table 7 Distributor's expected effective output under different demand quantities

\begin{tabular}{|c|c|c|c|c|c|c|c|c|c|c|c|c|c|c|c|c|c|c|}
\hline & \multicolumn{2}{|c|}{0.1} & \multicolumn{2}{|c|}{0.2} & \multicolumn{2}{|c|}{0.3} & \multicolumn{2}{|c|}{0.4} & \multicolumn{2}{|c|}{0.5} & \multicolumn{2}{|c|}{0.6} & \multicolumn{2}{|c|}{0.7} & \multicolumn{2}{|c|}{0.8} & \multicolumn{2}{|c|}{0.9} \\
\hline$Q$ & $T P D$ & $\hat{s}$ & $T P D$ & $\hat{s}$ & $T P D$ & $\hat{s}$ & $T P D$ & $\hat{s}$ & $T P D$ & $\hat{s}$ & $T P D$ & $\hat{s}$ & $T P D$ & $\hat{s}$ & $T P D$ & $\hat{s}$ & $T P D$ & $\hat{s}$ \\
\hline 50 & 5936 & 14.9 & 6186 & 17.1 & 6460 & 19.7 & 6750 & 22.7 & 7093 & 26.9 & 7543 & 33.6 & 8177 & 48.7 & 8992 & 145.6 & 9775 & $\infty$ \\
\hline 150 & 25654 & 6.4 & 25912 & 8.1 & 26154 & 9.7 & 26369 & 11.3 & 26594 & 13.1 & 26860 & 15.4 & 27224 & 18.9 & 27840 & 26.1 & 29326 & 74.3 \\
\hline 350 & 64779 & 0 & 65927 & 0 & 66469 & 0.08 & 66780 & 2 & 67058 & 3.8 & 67338 & 5.7 & 67665 & 8.1 & 68130 & 12 & 69179 & 23.6 \\
\hline
\end{tabular}




\section{Conclusion}

This paper has mainly studied the issues of a complex supply chain system scheduling approach based on the general conditions of TOC. As in the fierce market competition, the most fierce market environment faced by the short life cycle product supply chain is the declining value of the product, continuous introduction of same series products and similar products from competitors. Therefore, how to establish a supply chain system in accordance with time-based competition through application of TOC based supply chain coordination methods, improve the whole supply chain competitive advantage under general condition and thus lead to higher profit margins have become major research topics of this chapter. For the specific research methods, this chapter has firstly through establishment of a SDBR based supply chain system, by using of the Make to availability as the premise in advance production for inventory and taking the manufacturer's production capacity and distributor's advance production quantity as specific decision variables, and then through modeling analysis, obtained the optimal decision conclusions of the manufacturer and distributor under different conditions.

\section{Acknowledgement}

This paper is sponsored by the National Science Foundation serial numbers 71402048 and 71403085 , and Research Center of Hubei logistics development, serial number 2016A01.

\section{References}

[1 ]Andel, T. (1996). Manage inventory, own information. Transportation \& Distribution, 37(5), 54-58.

[2] Buchmeister, B., Friscic, D., Lalic, B., \& Palcic, I. (2012). Analysis of a three-stage supply chain with level constraints. International Journal of Simulation Modelling,11(4), 196-210.

[3] Chatfield, D. C., \& Pritchard, A. M. (2013). Returns and the bullwhip effect. Transportation Research Part E: Logistics and Transportation Review, 49(1), 159-175.

[4] Chen, F., Drezner, Z., Ryan, J. K., \& Simchi-Levi, D. (2001). Quantifying the bullwhip effect in a simple supply chain: The impact of forecasting, lead times, and information. Management Science, 46(3), 436-443.

[5] Chen, L., \& Lee, H. L. (2012). Bullwhip effect measurement and its implications. Operations Research, 60(4), 771-784.

[6] Cole, H., \& Jacob, D. (2003). Introduction to TOC supply Chain. AGI Institute.

[7] Cox, J. F., \& Spencer, M. S. (1998). The constraints management handbook. Boca Raton, FL: Lucie 
Press.

[8] De la Fuente, D., \& Lozano, J. (2007). Application of distributed intelligence to reduce the bullwhip effect. International Journal of Production Research, 44(8), 1815-1833.

[9] Disney, S. M., Farasyn, I., Lambrecht, M., Towill, D. R., \& Van de Velde, W. (2005).Taming the bullwhip effect whilst watching customer service in a single supply chain echelon. European Journal of Operational Research, 173(1), 151-172.

[10] Disney, S. M., \& Towill, D. R. (2003). On the bullwhip and inventory variance produced by an ordering policy. Omega -The International Journal on Management Science., 31, 157-167.

[11] Forrester, J. W. (1961). Industrial dynamics. Cambridge, MA: MIT Press.

[12] Fox, M. S., Chionglo, J. F., \& Barbuceanu, M. (1993). The integrated supply chain management system. Internal Report, Dept. of Industrial Engineering, University of Toronto.

[13] Goldratt, E. M. (1990). Theory of constraints. Croton-on-Hudson, NY: North River Press.

[14] Goldratt, E. M., \& Cox, J. (1984). The goal-A process of ongoing improvement. Crotonon- Hudson, NY: North River Press.

[15] Jose C., Borja P., David D.L. F., Raul. P. Julio P..(2015) Applying Goldratt's theory of constraints to reduce the bullwhip effect through agent-based modeling. Expert System with Applications, 42, 2049-2060

[16] Liang, W. Y., \& Huang, C. C. (2006). Agent-based demand forecast in multi-echelon supply chain. Decision Support Systems, 42(1), 390-407.

[17] Lengkaijun , Chen Xiangjun.((2012) .A Genetic Algorithm for TOC-based Supply Chain Coordination . Applied Mathiematics \& Information Sciences, 6(3), 67-774

[18] Maturana, F., Shen, W., \& Norrie, D. H. (1999). MetaMorph: An adaptive agent-based architecture for intelligent manufacturing. International Journal of Production Research, 37(10), 2159-2173.

[19] Saberi, S., Nookabadi, A. S., \& Hejazi, S. R. (2012). Applying agent-based system and negotiation mechanism in improvement of inventory management and customer order fulfillment in multi echelon supply chain. Arabian Journal for Science and Engineering, 37(3), 851-861.

[20] Simatupang, T. M., Hurley, S. F., \& Evans, A. N. (1997). Revitalizing TQM efforts: a self-reflection diagnosis based on the theory of constraints. Management Decision, 35(10), 746-752.

[21] Simatupang, T. M., Wright, A. C., \& Sridharan, R. (2004). Applying the theory of constraints to supply chain collaboration. Supply Chain Management: An International Journal, 9(1), 57-70.

[22] Sterman, J. D. (1989). Modeling managerial behavior: Misperceptions of feedback in a dynamic decision making experiment. Management Science, 35(3), 321-339.

[23] Umble, M., Umble, E., \& von Deylen, L. (2001). Integrating enterprise resources planning and theory of constraints: A case study. Production and Inventory Management Journal, 42(2), 43-48.

[24] Wu, H. H., Chen, C. P., Tsai, C. H., \& Tsai, T. P. (2010). A study of an enhanced simulation model for TOC supply chain replenishment system under capacity constraint. Expert Systems with Applications, 37, 6435-6440.

[25] Wu, H. H., Lee, A. H. I., \& Tsai, T. P. (2014). A two-level replenishment frequency model for TOC supply chain replenishment systems under capacity constraint.Computers \& Industrial Engineering, 72, 152-159.

[26] Youngman, K. (2009). A guide to implementing the theory of constraints (TOC). $<$ http://www.dbrmfg.co.nz/>. Last access 9 July 2014.

[27] Yuan, K. J., Chang, S. H., \& Li, R. K. (2003). Enhancement of theory of constraints replenishment 
using a novel generic buffer management procedure. International Journal of Production Research, 41(4), 725-740.

[28] Zarandi, M. H., Fazel Pourakbar, M., \& Turksen, I. B. (2008). A fuzzy agent-based model for reduction of bullwhip effect in supply chain systems. Expert Systems with Applications, 34(3), 1680-1691. 